\title{
Pericardial Effects on Diastolic Ventricular Interaction during Development
}

\author{
B. M. MINCZAK, M. R. WOLFSON, W. P. SANTAMORE, AND T. H. SHAFFER
}

Departments of Physiology and Pediatrics, Temple University School of Medicine, Philadelphia, Pennsylvania 19140, and Department of Cardiology, Bowman Gray School of Medicine, Winston-Salem, North Carolina 27103

\begin{abstract}
The effects of the pericardium on myocardial diastolic ventricular interaction during early development were determined in vitro using hearts excised from eight preterm $(109 \pm 0.106 \mathrm{SE} d$ gestation; term $=147 \mathrm{~d})$ and eight newborn $(2.3 \pm 0.30 \mathrm{SE}$ d postnatal age) lambs. The lamb hearts were excised with the pericardium intact and immersed in a cold cardioplegic solution. Subsequently, very compliant balloon catheters were inserted into the right and left ventricles retrograde through the pulmonary artery and aorta respectively. Before and after the removal of the pericardium, the changes in left ventricular pressure or volume caused by increasing right ventricular pressure or volume were measured. Computerized analysis of the pressure and volume recordings yielded pressure and volume transfer functions that quantified ventricular interaction. The pressure transfer functions (left ventricular pressure/right ventricular pressure) both with and without the pericardium intact were $0.297 \pm 0.010 \mathrm{SE}$ and $0.140 \pm$ $0.005 \mathrm{SE}$, respectively, for the preterms and $0.650 \pm 0.033$ $\mathrm{SE}$ and $0.301 \pm 0.031 \mathrm{SE}$ for the newborns. The volume transfer functions (left ventricular volume/right ventricular volume) with and without the pericardium were $0.320 \pm$ $0.04 \mathrm{SE}$ and $0.150 \pm 0.024 \mathrm{SE}$, respectively, for the preterm and $0.514 \pm 0.052 \mathrm{SE}$ and $0.233 \pm 0.027 \mathrm{SE}$ for the newborns. These data demonstrate that 1 ) the pericardium increases ventricular interaction in both preterm and newborn lambs and 2) the relative percentage increase is similar for both age groups and not age dependent. Furthermore, these findings suggest that the increase in ventricular interaction due to the presence of the pericardium is mediated by the relative compliances of the free walls, septum, and pericardium. (Pediatr Res 27: 547-551, 1990)
\end{abstract}

\begin{abstract}
Abbreviations
dpl, derivative of left ventricular pressure dvl, derivative of left ventricular volume dpr, derivative of right ventricular pressure dvr, derivative of right ventricular volume
\end{abstract}

The ventricles of the heart are in close anatomical proximity and share a common deformable wall, the interventricular septum. Because of this structural configuration, perturbations of volume or pressure in one ventricle are capable of effecting changes in the pressure-volume relationship of the contralateral ventricle. This phenomenon of transferring pressure and/or volume changes from one ventricle to the opposite ventricle directly

Received April 3, 1989, accepted January 16, 1990

Correspondence and reprint requests: B. M. Minczak, Ph.D., c/o T. H. Shaffer, Ph.D., Department of Physiology, Temple University School of Medicine, 3420 North Broad Street, Philadelphia, PA 19140.

Supported in part by NIH \#HL36068 and AHA 584-424-01. through the myocardium, independent of neural, circulatory, or humoral effects, is termed ventricular interaction (1-3).

Previous in vitro investigations have demonstrated age-related differences in diastolic ventricular interaction in the absence of the pericardium (4). These differences in interaction were attributed to a difference in the relative compliances of the interventricular septae between preterm and newborn lamb hearts. In a previous study (4), no difference was demonstrated between the free wall compliances of the right and left ventricular free walls of either group. The effect of the developing pericardium on ventricular interaction is not known. Studies of adult hearts enclosed by the pericardium and having a relatively more compliant right than left ventricular free wall have indicated that the pericardium enhances ventricular coupling (5-18). Furthermore, other adult studies suggest that the pericardium may grow or adapt to increased volume loads imposed on the myocardium (17, 19-21). Myocardial development may actually stimulate growth and affect endogenous characteristics of the pericardium (22-24). Therefore, it is reasonable to postulate that the pericardium may affect ventricular coupling in developing hearts and that these effects may vary at different time points in development (25-29).

Our first objective was to determine the effect of the pericardium on ventricular interaction in preterm and newborn lamb hearts. In addition, based on a comparison of the data from both groups, we have attempted to describe the effects of the pericardium on ventricular interaction with respect to development. Currently, there are no data available that directly address the effects of the pericardium on ventricular interaction during early development.

\section{MATERIALS AND METHODS}

Animal Preparation. The animals used in this experiment were divided into two groups: the first group was made up of eight preterm lambs $(109 \pm 0.106 \mathrm{SE} d$ gestation; term $=147 \mathrm{~d})$ weighing $1.89 \pm 0.07 \mathrm{SE} \mathrm{kg}$ with heart wt of $14.23 \pm 0.27 \mathrm{SE} \mathrm{g}$, delivered by hysterotomy. The mean free wall and septal wt of the hearts in this group were right free wall $=5.34 \pm 1.2 \mathrm{SE} \mathrm{g}$, left free wall $=5.75 \pm 1.85 \mathrm{SE} \mathrm{g}$, septum $=3.12 \pm 1.25 \mathrm{SE} \mathrm{g}$. The second group was comprised of eight newborn lambs $(2.3 \pm$ $0.30 \mathrm{SE} d$ postnatal age, weighing $3.4 \pm 0.11 \mathrm{SE} \mathrm{kg}$, with heart wt of $26.3 \pm 1.1 \mathrm{SE} \mathrm{g}$ ) previously delivered vaginally. The mean free wall and septal wt of this group were right free wall $=8.64$ $\pm 1.65 \mathrm{SE} \mathrm{g}$, left free wall $=9.11 \pm 1.35 \mathrm{SE} \mathrm{g}$, septum $=8.55 \pm$ $1.33 \mathrm{SE} g$.

Ketamine hydrochloride $10 \mathrm{mg} / \mathrm{kg}$ body wt intramuscularly was given to the pregnant ewes, which were used for obtaining the preterm lambs, before induction of spinal anesthesia with $4 \%$ bupivicaine epidurally.

A lethal dose of magnesium chloride $(50 \mathrm{mg} / \mathrm{kg}$ body wt i.v.) was given to the lambs in both groups, euthanizing them. Subsequently, their hearts, with the pericardium intact, were immediately excised, via a mid-sternal thoracotomy. The hearts were 
then placed in a cold $\left(10^{\circ} \mathrm{C}\right)(30)$ cardioplegic solution (sodium chloride $602 \mathrm{mg} / 100 \mathrm{~mL}$, calcium chloride $23.1 \mathrm{mg} / 100 \mathrm{~mL}$, potassium chloride $119.3 \mathrm{mg} / 100 \mathrm{~mL}$, sodium bicarbonate 37 $\mathrm{mg} / 100 \mathrm{~mL}$, mannitol $1250 \mathrm{mg} / 100 \mathrm{~mL}$, dextrose $300 \mathrm{mg} / 100$ $\mathrm{mL}$ ). This was performed to prevent any active tension development and retard postmortem deterioration (4, 30, 31). Retroperfusion of the coronary arteries with cardioplegic solution was achieved via a small lumen catheter, which was inserted into the coronary ostia at the aortic root. This was performed to further attenuate postmortem compliance and metabolic changes. Subsequently, very compliant balloon catheters fabricated from balloons attached to rigid polyethylene 240 (inner diameter $0.066^{\prime \prime}$; outer diameter $0.095^{\prime \prime}$ ) tubing were inserted into the right and left ventricles. To alleviate any significant back pressure development, the balloons were previously overinflated $(100 \mathrm{~mL})$ with water for several days. The balloon catheters were deflated and placed retrograde via the pulmonary artery and aorta into the right and left ventricles, respectively, and ligated into place. To ensure proper balloon contact with the myocardial walls, the chordae tendinae were severed and a visual inspection of the heart was performed as cold water was infused and withdrawn via a syringe into the catheterized ventricles. The balloon catheters were then filled with an "unstressed" volume (i.e. producing a pressure of zero, right ventricular vol $=5 \pm 1 \mathrm{SE} \mathrm{mL}$; left ventricular vol $=4 \pm 0.9 \mathrm{SE} \mathrm{mL}$ ) of cold water. The catheter tip within the balloon was approximated to the middle of the ventricle. The instrumented heart was then connected to a manifold of stopcocks. This manifold joined an infusion pump (Harvard Apparatus Co., Inc., S. Natick, MA), to the right ventricular balloon and pressure transducer (Statham P23db, Perez, Puerto Rico). The left ventricular balloon was also joined to a pressure transducer. To measure volume changes, a collection chamber was hung from a force transducer (Biocom, Culver City, CO) calibrated to yield volume for a given wt change (Fig. 1). Simultaneous pressure and volume measurements were made in the ventricles as the volume of one ventricle was varied.

Experimental protocol. The effects of the pericardium on ventricular interaction were determined by performing the subsequent protocol $(4,30)$ before and after the removal of the pericardium. A set of two measurement runs were made, one with the responding ventricular volume constant (vol range $=4$ $\pm 0.3 \mathrm{SE} \mathrm{mL}$ ), i.e. isovolumic, and the other with the responding ventricular pressure constant $(3-5 \mathrm{~mm} \mathrm{Hg})$ or isobaric, allowing the responding ventricle to eject volume. In the first run, the stopcock (Fig. 1) was closed, preventing the left or "responding"

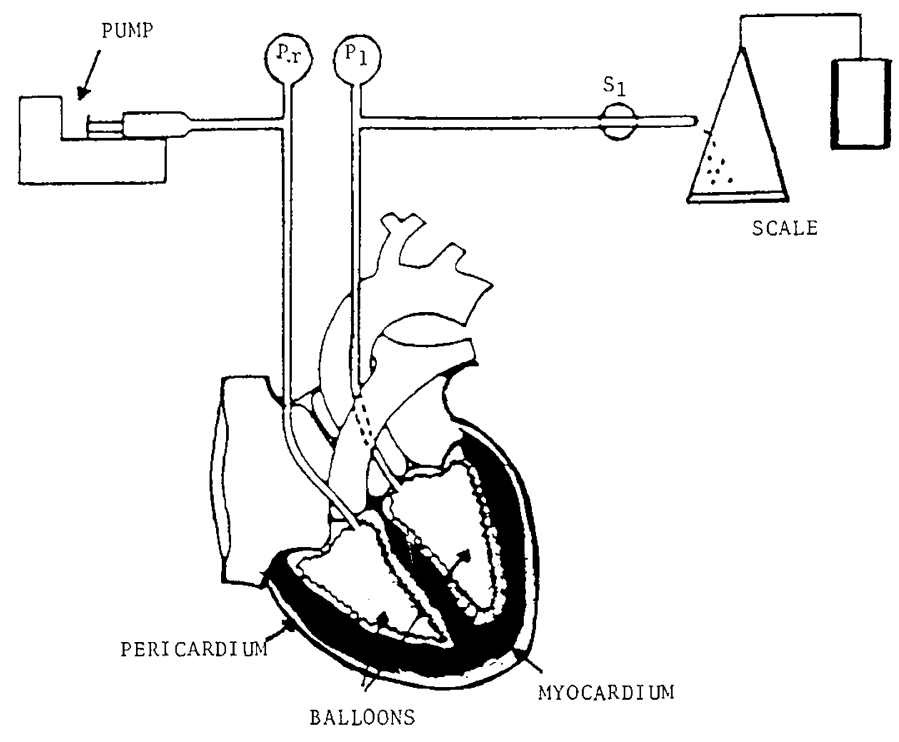

Fig. 1. Experimental set-up. Components: infusion pump (Harvard Apparatus Co., Inc.), pressure transducers $(P)$, balloon catheterized heart, volume collection chamber, scale, force transducer $(S 1)$ stopcock. ventricular volume from changing. The infusion pump was set to a known flow rate $(3.9 \mathrm{~mL} / \mathrm{min})$ and activated, infusing cold water into the right or "driving" ventricle. Simultaneous right and left ventricular pressures were measured during the infusion by the respective pressure transducers. An Electronics for Medicine (White Plains, NY) VR6 recorder was used to measure the right and left ventricular pressure changes. The right ventricular volume change as a result of the infusion was determined by calculating the product of the known infusion rate and the duration of the infusion. Time marks on the hardcopy trace were used to determine the duration of the infusion.

During the second run, the stopcock (Fig. 1) was opened, allowing left ventricular volume to change while maintaining left ventricular pressure constant $(3-5 \mathrm{~mm} \mathrm{Hg}$ ). Once again the infusion pump was activated, but now the left ventricular volume changed. As the right ventricular pressure and volume increased, cold water was ejected from the left ventricular catheter into the volume collection chamber. This volume change and the concomitant right ventricular pressure change were also recorded. The concomitant right ventricular volume change was determined as in run 1. Each run was repeated two times. Upon completion of the preceding protocol, the pericardium was removed and the measurements repeated. It took 45 to $60 \mathrm{~min}$ to complete the entire experimental protocol. The stability of the preparation was tested at the end of the experimental protocol to determine if differences occurred in the transfer functions as a function of postmortem time. This was accomplished by repeating the initial pressure transfer function measurements and comparing the results to those obtained immediately postremoval of the pericardium. No significant differences were observed in the data. Furthermore, the $\mathrm{pH}$ and temperature $\left(10^{\circ} \mathrm{C}\right)$ of the cardioplegic solution remained constant for the duration of the experimental protocol.

Computerized data analysis. Using a computer (Apple-II Plus, Cupertino, CA) and an Apple digitizing pad with cross-haired cursor, the above data were analyzed $(4,30,32)$. Upon tracing the analog pressure and volume records with the cursor, the computer converted these signals into digital pressure and volume values. The computer software accomplished this by referencing the pressure and volume calibration signals of $30 \mathrm{~mm} \mathrm{Hg}$ and $1 \mathrm{~mL}$ full scale, respectively. These were input at the beginning of the experiment.

Transfer function data was calculated by using a specifically designed computer program $(4,30)$. Using a sliding 2 nd-order polynomial fit $(4,30,32)$, derivatives of the pressure and volume changes were calculated and appropriately divided point by point to yield transfer functions (dpl/dpr, dvl/dvr).

Transfer function determinations. The degree of interaction between the myocardial ventricles was quantified by two previously described transfer function values $(4,30)$. The pressure transfer $(\mathrm{dpl} / \mathrm{dpr})$ that quantifies a left ventricular pressure change during a concomitant right ventricular pressure change was determined from measurements during infusion of the right ventricle while the left ventricular volume $(3.4 \pm 0.4 \mathrm{SE} \mathrm{mL})$ was held constant (run 1). The volume transfer function (dvl/ $\mathrm{dvr}$ ) was determined from measurements during right ventricular infusion while the left ventricular pressure was held constant (3$5 \mathrm{~mm} \mathrm{Hg}$ ) (run 2).

Statistical considerations. Pressure and volume transfer functions both with and without the pericardium were evaluated via a single-tailed $t$ test to determine if there was a significant increase in pressure and volume transfer with the pericardium intact. All transfer function values reported were an average at a given driving ventricular pressure of $5.5 \mathrm{~mm} \mathrm{Hg}$. In addition, the effect of age on the transfer functions was determined using an analysis of covariance, i.e. age and transfer functions with and without the pericardium. A logarithmic transformation was made of the percent transfer function change to normalize the populations and provide a more sensitive test of the relationship. To test if the pericardium affects the transfer of pressure and volume 
information in the two age groups differently, a paired $t$ test with logarithmic transformation was performed on the percent differences with the pericardium present in both groups.

\section{RESULTS}

In Figure 2, typical data traces with and without the pericardium are depicted. As is evident from the figure, the presence of the pericardium causes an increase in the slopes of the responding ventricular pressure and volume curves. These responses were computer analyzed to yield the pressure and volume transfer functions. Pressure transfer function data obtained with and without the pericardium for the preterm and newborn groups are represented in Figure 3. As shown in Figure 3, pressure transfer between the ventricles of the preterm group $(\mathrm{dpl} / \mathrm{dpr}=$ $0.297 \pm 0.01 \mathrm{SE}$ ) was greater with the pericardium intact than without the pericardium $(\mathrm{dpl} / \mathrm{dpr}=0.140 \pm 0.005 \mathrm{SE})(p<$ 0.01 ). With respect to the newborn group, pressure transfer between the ventricles of newborn hearts with the pericardium intact was also greater $(\mathrm{dpl} / \mathrm{dpr}=0.650 \pm 0.033 \mathrm{SE})$ than without the pericardium $(\mathrm{dpl} / \mathrm{dpr}=0.301 \pm 0.031 \mathrm{SE})(p<0.01)$. The pressure transfer both with and without the pericardium intact was greater $(p<0.05)$ in the newborns than in the preterm hearts.
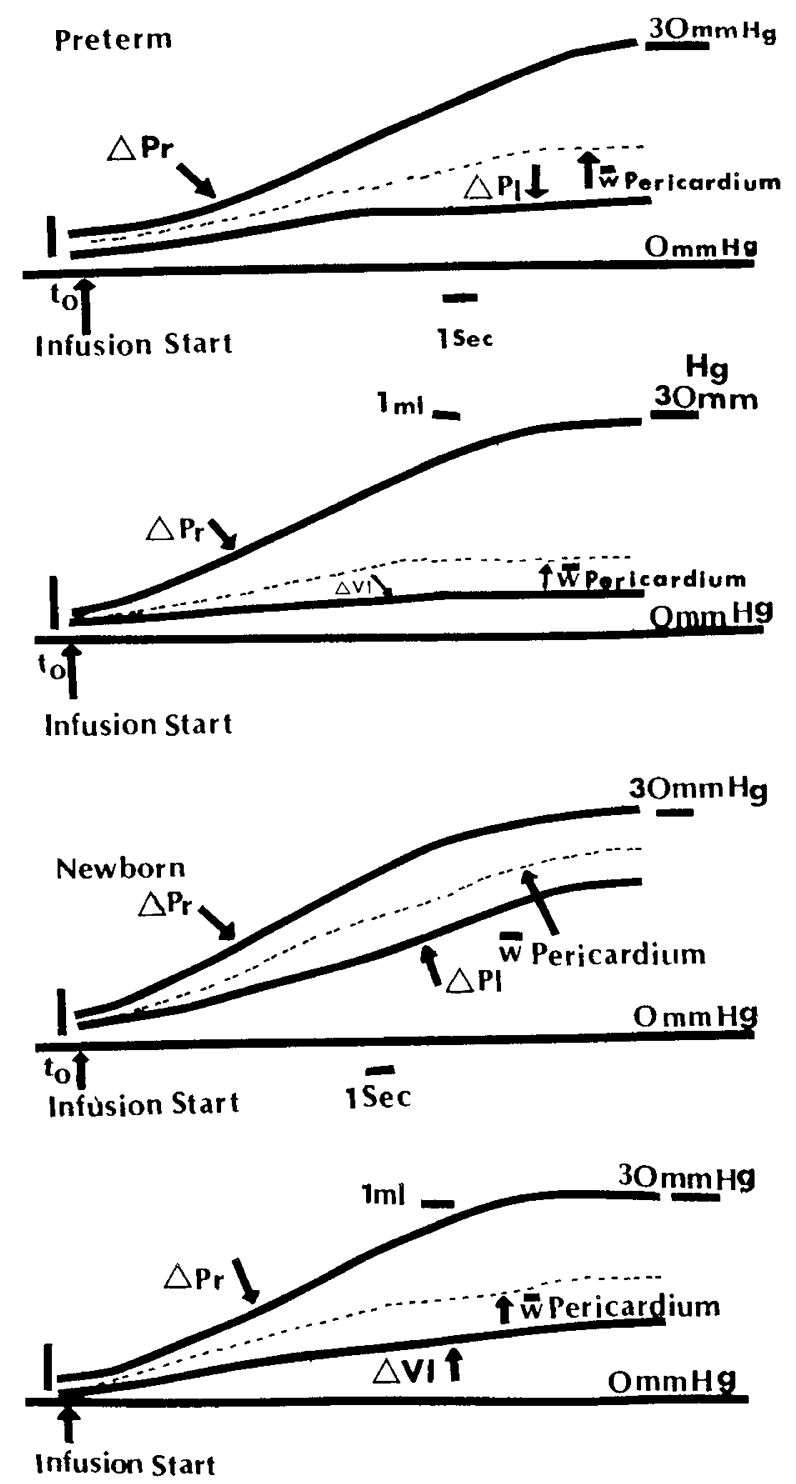

Fig. 2. Schematic of real data traces. Upper panel depicts preterm $\mathrm{dpl} / \mathrm{dpr}$ and $\mathrm{dvl} / \mathrm{dvr}$. Lower panel depicts pressure and volume transfer functions for the newborns.

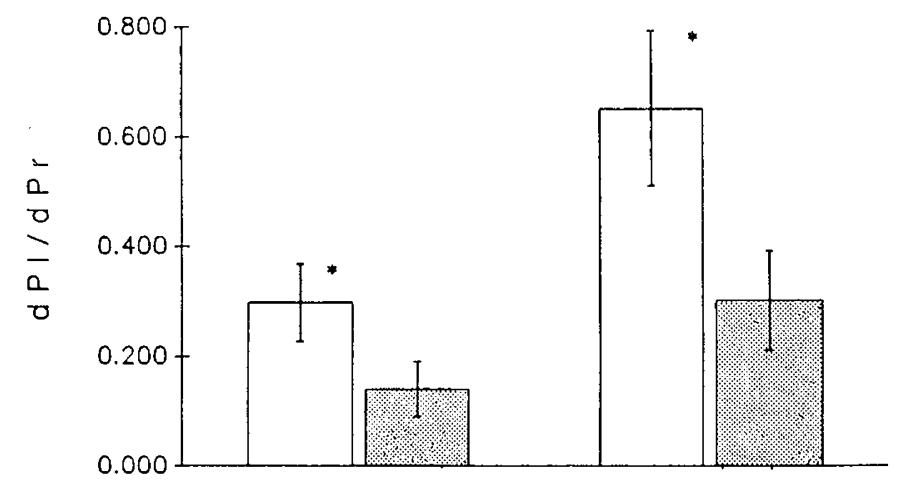

PRETERM HEARTS

NEWBORN HEARTS

Fig. 3. Pressure transfer functions $(\mathrm{dpl} / \mathrm{dpr})$ with and without the pericardium; preterm and newborn groups (open, with pericardium; stippled, without pericardium; $\left.{ }^{*}=p<0.05\right)$. Bars $=\mathrm{SE}$.

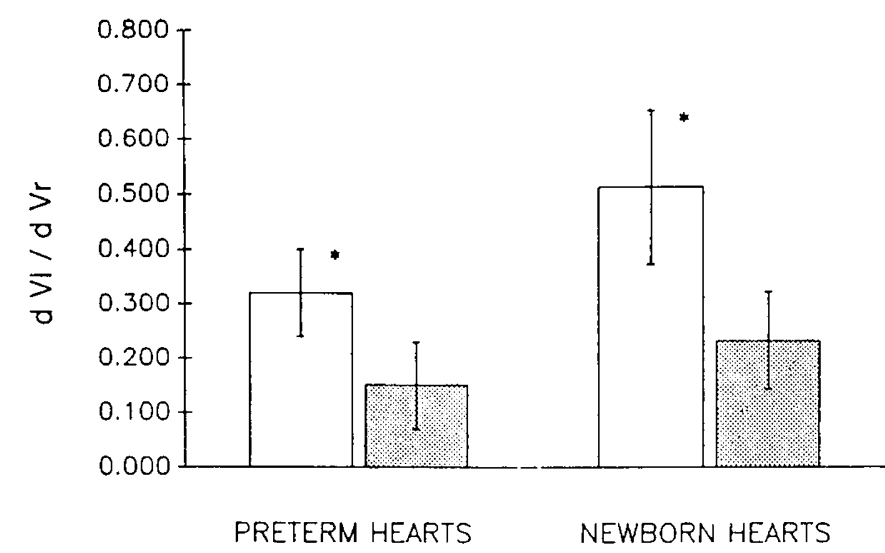

Fig. 4. Volume transfer functions ( $\mathrm{dvl} / \mathrm{dvr}$ ) with and without the pericardium; preterm and newborn groups (open, with pericardium; stippled, without pericardium; $\left.{ }^{*}=p<0.05\right)$. Bars $=\mathrm{SE}$.

Table 1. Percent change in transfer functions due to presence of pericardium (Mean $\pm S E$ )

\begin{tabular}{lcc}
\hline \multicolumn{1}{c}{$\begin{array}{c}\text { Pericardial } \\
\text { effect }\end{array}$} & Preterm & Newborn \\
\hline $\begin{array}{l}\text { Pressure transfer func- } \\
\text { tions (\% change dpl/ } \\
\text { dpr) }\end{array}$ & $114.4 \pm 3.94$ & $128.9 \pm 7.85$ \\
& & \\
Volume transfer func- \\
tions (\% change dvl/ \\
dvr)
\end{tabular}

Figure 4 depicts the volume transfer functions (dvl/dvr) for the preterm and newborn hearts both with and without the pericardium intact. The presence of the pericardium increased the degree of interaction in the preterm hearts $(\mathrm{dvl} / \mathrm{dvr}=0.320$ $\pm 0.04 \mathrm{SE})$ versus the preterm hearts without the pericardium $(\mathrm{dvl} / \mathrm{dvr}=0.150 \pm 0.024 \mathrm{SE})$. Likewise, the presence of the pericardium significantly increased $(p<0.01)$ the volume coupling between the ventricles of the newborn hearts, with the pericardium $(\mathrm{dvl} / \mathrm{dvr}=0.514 \pm 0.52 \mathrm{SE})$ versus without the pericardium $(\mathrm{dvl} / \mathrm{dvr}=0.233 \pm 0.027 \mathrm{SE})$. The volume transfer between the ventricles of the newborn was greater than that for the preterm $(p<0.01)$.

Table 1 summarizes the analysis of the relative effects of the pericardium on the two age groups. As shown, the pericardium causes a similar percentage increase in the pressure and volume transfer between the ventricles of both groups. 


\section{DISCUSSION}

Our study expanded our previous observations of diastolic ventricular interdependence in the developing heart by examining the influence of the pericardium on mechanical coupling between the ventricles (4). The results demonstrate that the pericardium increased pressure and volume transfer between the ventricles in both preterm and newborn lamb hearts. Although the effects of the mediastinum and lungs (33) were obviated due to the in vitro nature of this preparation (30), this study has given us insight into the effects of the pericardial sac (per se) on ventricular interaction.

Several experimental studies in adult hearts have observed that the pericardium increased the pressure and volume transfer between the ventricles. Glantz et al. (11) showed that with the pericardium intact, right ventricular pressure was a better predictor of left ventricular pressure than were left ventricular dimensions. Using an isolated heart preparation, Janicki and Weber (12) showed that the coupling between the ventricles was significantly greater with the pericardium intact, particularly for larger end diastolic volumes. Their data suggested that the pericardium may have a restricting effect on the myocardial free walls of adult hearts. Other studies of adult hearts have, in fact, demonstrated that the pericardium does have a restrictive effect on overdistension of the myocardium $(5-8,11,34)$. In addition to experimental studies, recent theoretical analyses of ventricular interdependence have shown that the mechanistic factors that affect ventricular interaction must include not only transeptal pressure gradients and free wall and septal compliances, but also a pericardial component (18).

In contrast to the extensive adult literature, little information regarding the effects of the pericardium on ventricular distensibility during early development is currently available. In addition, the studies that were previously done only describe whole right and left ventricular pressure-volume curve shifts, indicating that the intraventricular pressure of the left ventricle has a pericardial component that increases at higher intraventricular volumes and/or pressures $(9,12,15,35)$. Unlike previous investigations, our study describes how the pericardium directly affects the pressure and volume information transfer between the ventricles, i.e. how the transfer functions $(4,30)$ are affected. With respect to both pressure (dpl/dpr) and volume $(\mathrm{dvl} / \mathrm{dvr})$ information transfer between the ventricles (Figs. 3 and 4), the effect of the pericardium is seen as an increase in pressure and volume information transfer during concomitant filling of both preterm and newborn lamb hearts. Although we are reporting the degree of ventricular interaction at $5.5 \mathrm{~mm} \mathrm{Hg}$ pressure, chosen to approximate physiologic conditions $(36,37)$, we feel that these findings suggest that the presence of the pericardium affects the overall distensibility of the right and left myocardial ventricles by restricting the potential displacement of the right and left ventricular free wall. It also follows that at higher intracardiac volumes or during pericardial distention, i.e. tamponade, the effects of the pericardium on ventricular interaction may be even greater. Furthermore, because myocardial tissue is nonlinearly elastic and the transfer functions vary nonlinearly at higher filling pressures of the driving ventricle (38), the effects of the pericardium at higher albeit unphysiologic/pathologic volumes and pressures may be quite different.

Previous work from our laboratory has described and verified a theoretical analysis of ventricular interaction on the basis of transseptal pressure gradients and the relative compliances of the ventricular free walls and interventricular septum $(4,35,39)$. Our current findings further substantiate this analytical model and previous observations regarding pressure-volume coupling (4). Furthermore, our current data strengthen our previous findings (4) based on the similarity of the transfer functions measured without the pericardium in both the preterm and newborn groups. In addition, we have found that the increase in the mechanical pressure-volume coupling of the myocardial ventri- cles attributed to the pericardium is similar in both the preterm and newborn. Table 1 shows no significant age-related differences, suggesting that the pericardium and the myocardium may be growing at a similar rate $(17,19,21)$.

The results of our study indicate increased coupling between the pericardial cavity and the ventricles, and observation confirmed by previous experimental and theoretical adult studies $(2$, $8,11,13)$. However, since the pericardial space is a potential rather than a real space, local differences in pericardial pressure may occur and not be transmitted from one ventricle to another (35). Thus, for the pericardial effects to be significant, filling of one ventricle would have to modify the transpericardial pressure (pericardial pressure minus pleural pressure). As indicated by previous investigators $(40,41)$, under normal circumstances, the volume change of one ventricle can modulate transpericardial pressure, but this effect is small and most coupling between the ventricles occurs through the myocardium. If the pericardium becomes very stiff, however, coupling would become greatly accentuated. This increased ventricular interdependence might explain some aspects of cardiac tamponade and constrictive pericarditis in which effective pericardial compliance has been decreased.

However, we must also state that our current findings must be accepted under the realization that the experiments were conducted at subphysiologic temperatures $\left(10^{\circ} \mathrm{C}\right)$. The protocol necessitates conducting the transfer function measurements at a cold temperature $\left(10^{\circ} \mathrm{C}\right)(20)$. If we were to run the experiments at $37^{\circ} \mathrm{C}$, the compliances of the free walls would change markedly in a relatively short period of time. This would cause an underestimation of the true compliances of the myocardial free walls and septum. To conduct these experiments at a more physiologic temperature, retroperfusion via the aortic root is necessary. These experiments are currently underway in beating, isolated heart preparations and will be presented in a later publication that will also encompass systolic ventricular interaction.

In conclusion, the role of the pericardium is not only to prevent the heart from overdistending, but also to increase the degree of ventricular interaction between the ventricles at early stages of development. In addition, the influence of the pericardium in preterm and newborn hearts is similar, suggesting that the pericardium may grow as the heart grows.

Acknowledgment. The authors thank Robert Roache for his expert technical assistance.

\section{REFERENCES}

1. Glantz SA, Parmley WW 1978 Factors which affect the diastolic pressurevolume curve. Circ Res 42:171-180

2. Janicki JS, Weber KT 1980 Factors influencing the diastolic pressure-volume relation of the cardiac ventricles. Fed Proc 39:133-140

3. Bove AA, Santamore WP 1981 Ventricular interdependence. Prog Cardiovasc Dis 23:365-388

4. Minczak BM, Wolfson MR, Santamore WP, Shaffer TH 1988 Development changes in diastolic ventricular interaction. Pediatr Res 23:466-469

5. Berglund E, Sarnoff SJ, Isaacs JP 1955 Ventricular function: Role of the pericardium in regulation of cardiovascular hemodynamics. Circ Res 3:133139

6. Hefner LL, Coghlan HC, Jones WB, Reeves TJ 1961 Distensibility of the dog left ventricle. Am J Physiol 201:97-101

7. Holt JP 1970 The normal pericardium. Am J Cardiol 26:455-465

8. Spotnitz HM, Kaiser GA 1971 The effects of the pericardium on pressurevolume relations in the canine left ventricle. J Surg Res 11:375-380

9. Elzinga G, Van Grondelle R, Westerhof N, Van Den Bos GC 1974 Ventricular interference. Am J Physiol 226:941-947

10. Shirato K Shabetai R, Bhargava V, Franklin D, Ross Jr J 1978 Alterations of the left ventricular diastolic pressure-segment length relation produced by the pericardium. Circulation 57:1191-1198

11. Glantz SA, Misbach GA, Moores WY, Mathey DG, Lekven J, Stowe DF, Parmley WW, Tyberg JV 1978 The pericardium substantially affects the left ventricular diastolic pressure-volume relationship in the dog. Circ Res 42:171-180

12. Janicki JS, Weber KT 1980 The pericardium and ventricular interaction, distensibility and function. Am J Physiol 238:H494-H503

13. Spadaro J, Bing OHL, Gaasch WH, Weintraub RM 1981 Pericardial modulation of right and left ventricular diastolic interaction. Circ Res 48:233-238 
14. Molaug M, Stokland O, Ilebekk A, Lekven J, Kiil F 1981 Myocardial function of the interventricular septum: effects of right and left ventricular pressure loading before and after pericardiectomy in dogs. Circ Res 49:52-61

15. Maughan WL, Kallman CH, Shoukas A 1981 The effect of right ventricular filling on the pressure-volume relationship of the ejecting canine left ventricle. Circ Res 49:382-388

16. Slinker BK, Glantz SA 1986 End-systolic and end-diastolic ventricular interdependence. Am J Physiol 1987 43:231-245

17. LeWinter MM, Pavelec R 1982 Influence of the pericardium on left ventricular end-diastolic pressure-segment relations during early and later stages of experimental chronic volume overload in dogs. Circ Res 50:501-509

18. Santamore WP, Shaffer TH, Papa L Theoretical model of ventricular interdependence: pericardial effects: Basic Res Cardiol 1987 83:426-443

19. Gault JH, Covell JW, Braunwald E, Ross Jr J 1970 Left ventricular performance following correction of free aortic regurgitation. Circ Res 42:773-780

20. McCollough WH, Covell JW, Ross Jr J 1972 Left ventricular dilation and diastolic compliance changes during chronic volume overloading. Circulation 54:943-951

21. LeWinter MM, Engler RL, Karliner JS 1980 Enhanced left ventricular shortening during chronic volume overload in conscious dogs. Am J Physiol 238:H 126-H133

22. Dawes GS, Mott JC, Widdicombe JG 1938 The foetal circulation in the lamb. J Physiol 126:563-587

23. Holt JB 1970 The normal pericardium. Am J Cardiol 26:455-465

24. Holt JB, EA Ahode, H Kines 1960 Pericardial and ventricular pressure. Circ Res 8:1171-1181

25. Keen EN 1955 The postnatal development of the human cardiac ventricles. $\mathbf{J}$ Anat (Lond) 89:484-502

26. Hort W 1966 The normal heart of the fetus and its metamorphosis in the transition period. In: Cassels DE (ed) The Heart and Circulation in the newborn Infant. Grune and Stratton, New York, pp 210-224

27. Emery JL, Macdonald MS 1960 The weight of the ventricles in the later weeks of intrauterine life. Br Heart J 22:563-570
28. Recavarren S, Avia-Stella J 1964 Growth and development of the ventricular myocardium from birth to adult life. Br Heart J 26:187-192

29. Dawes GS 1968 Foetal and Neonatal Physiology. Yearbook Medical Publishers Inc, Chicago, pp 160-177

30. Santamore WP, Shaffer TH, Hughes DA 1986 Theoretical and experimental model of ventricular interdependence. Basic Res Cardiol 81:529-537

31. Rosenkranz ER, Buckberg GD 1983 Myocardial protection during surgical coronary reperfusion. J Am Coll Cardiol 1:1235-1246

32. Marble AE, McIntyre CM, Hastings-James R, Hor CW 1981 A comparison of digital algorithms used in computing the derivative of the left ventricular pressure. IEEE Trans Biomed Eng 28:524-528

33. Robatham JL, Mitzner W 1979 A model of the effects of respiration on left ventricular performance. I Appl Physiol 46:411-418

34. Maughan WL, Sunagawa K, Sagawa K 1987 Ventricular systolic interdependence: volume elastance model in isolated canine hearts. Am J Physiol 253:H1381-H1390

35. Mirsky I, Rankin JS 1979 The effects of geometry, elasticity, and external pressures on the diastolic pressure-volume and stiffness-stress relations. How important is the pericardium? Circ Res 44:601-611

36. Arcilla RA, Oh W, Lind J, Blankenship W 1966 Portal and atrial pressures in the newborn period. Acta Paediatr Scand 55:615

37. Anderson PAW, Manring A, Glick KL, Crenshaw Jr CL 1982 Biophysics of the developing heart III. A comparison of the ventricular dynamics of the fetal and neonatal heart. Am J Obstet Gynecol 143:195-205

38. Minczak BM 1987 Developmental changes in ventricular interaction during development: a theoretical and experimental approach. PhD thesis, Temple University, Philadelphia, PA

39. Santamore WP, Constantinescu M, Shaffer $\Upsilon 1988$ Predictive changes in ventricular interdependence. Ann Biomed Eng 16:215-234

40. Samiseth OA, Frais MA, Kingma I, Smith ER, Tyberg JV 1905 Assessment of pericardial constraint in dogs. Circulation 71:158-164

41. Tyson Jr GS, Maier GW, Olson CO, Davis JW, Rankin JS 1984 Pericardial influences on ventricular filling in the conscious dog. An analysis based on pericardial pressure. Circ Res 54:173-184 